\title{
Time to stop mammography screening?
}

\author{
Peter C. Gøtzsche MD
}

See related guidelines by the Canadian Task Force on Preventive Health Care on page 1991 and at www.cmaj.ca/lookup/doi/10.1503/cmaj.110334

$\mathrm{T}$ he Canadian Task Force on Preventive Health Care should be congratulated for its new recommendations on screening for breast cancer in women at average risk aged 40-74 years. ${ }^{1}$ These guidelines are more balanced and more in accordance with the evidence than any previous recommendations.

The recommendations against routine clinical breast examinations, breast self-examinations and magnetic resonance imaging to screen for breast cancer in this age and risk group are all straightforward.

The recommendations on mammography screening are even more conservative than the change in policy suggested by the US Preventive Services Task Force in 2009, which created an uproar in the United States from people interested in maintaining the status quo. The new Canadian guidelines are appropriately cautious, advising against routinely screening women aged 40-49 years. The task force recommends screening women aged 50-69 years every two to three years, although it admits that this is a weak recommendation based on moderate-quality evidence, and screening women aged 70-74 years on the same schedule based on low-quality evidence. The task force also suggests that women who do not place a high value on a small reduction in breast cancer mortality, and who are concerned with false-positive results on mammography and overdiagnosis, may decline screening.

These guidelines are an important step in the right direction, away from the prevailing attitude that a woman who does not undergo screening is irresponsible. Recent research even suggests that it may be most wise to avoid screening altogether, at any age, as outlined below.

The Canadian Task Force on Preventive Health Care decided not to include observational studies in its systematic review unless they were needed to elucidate the harms of screening or the values and preferences of patients. However, important observational studies have been published in recent years, without which a systematic review would be incomplete. ${ }^{2}$ These observational studies have been discussed elsewhere ${ }^{2}$ and have also been included in an update (cur- rently submitted for publication) of our 2009 Cochrane review of mammography screening. ${ }^{3}$

\section{Doubtful effect of screening}

If screening does not reduce the occurrence of advanced cancers, it does not work. A systematic review of studies from seven countries showed that, on average, the rate of malignant tumours larger than 20 millimetres was not affected by screening. ${ }^{2}$ Because the size of a tumour is linearly correlated to the risk of metastasis, ${ }^{4}$ this result is evidence against an effect of screening.

Denmark has a unique control group within its population - only $20 \%$ of its population was screened during a 17 -year period. The annual decrease in breast cancer mortality in the relevant age group (55-74 years) and period was $1 \%$ in the areas with screening and $2 \%$ in the nonscreened areas. ${ }^{5}$ Among women who were too young to benefit from screening, the decreases were larger (5\% for screened areas, $6 \%$ for unscreened areas). Similar results have been reported from the United Kingdom, Sweden and Norway.

A study involving women from 30 European countries showed that the mean decrease in breast cancer mortality between 1989 and 2005 among women less than 50 years of age was $37 \%$; the corresponding decrease was $21 \%$ among women aged 50-69 years. ${ }^{6}$ The declines began before the start of organized screening programs in many countries and are more likely explained by the introduction of tamoxifen. The introduction of tamoxifen could explain the
Competing interests: None declared.

This article was solicited and has not been peer reviewed.

Correspondence to: Dr. Peter C. Gøtzsche, pcg@cochrane.dk

CMAJ 2011. DOI:10.1503 /cmaj.111721 
larger decline seen among young women who often have estrogen-sensitive tumours.

Another study compared three pairs of similar neighbouring countries that had introduced screening 10-15 years apart. The pairs were Northern Ireland and the Republic of Ireland, the Netherlands and Belgium, and Sweden and Norway. There was no relation between start of screening and the reduction in breast cancer mortality. ${ }^{7}$ The fall in breast cancer mortality was about the same in all countries. Furthermore, the decline was also about the same as that seen in the United States, where screening started as early as in Sweden. ${ }^{8}$

Screening seems to be ineffective in today's world for two reasons. First, adjuvant therapy, such as tamoxifen and chemotherapy, is highly effective (even when the cancer has metastasized) but was not often used at the time of the old trials. Second, public awareness of breast cancer has increased, and women tend to see a doctor much earlier today when they have noticed something unusual in their breast. In Denmark, the average size of a tumour decreased by nine millimetres from 1979 to $1989,{ }^{2}$ a reduction that occurred before screening started. In addition, this decrease was larger than the average difference in tumour size seen between screened and control groups in trials $(5 \mathrm{~mm})$, despite the tendency for small, overdiagnosed tumours to spuriously exaggerate the difference. ${ }^{2}$

It has often been claimed that mammography screening reduces breast cancer mortality by $30 \% .{ }^{9}$ However, thorough systematic reviews have estimated only a $15 \%$ reduction, ${ }^{2,3}$ and data on tumour size from the trials are compatible with only a $12 \%$ effect. ${ }^{4}$ This effect is similar to the results seen in the most reliable studies, which showed a $10 \%$ effect after 13 years. ${ }^{3}$

\section{Overdiagnosis}

Any possible effect of screening on breast cancer mortality must be marginal and could be counteracted by the life-shortening effect that radiotherapy and chemotherapy have when used in healthy women in whom breast cancer has been overdiagnosed (i.e., a diagnosis of breast cancer that would not have been made in the woman's remaining life had she not undergone screen- ing). ${ }^{3}$ The main effect of screening is to produce patients with breast cancer from among healthy women who would have remained free of breast disease for the rest of their lives had they not undergone screening. Compelling data from the US, Norway and Sweden show that most overdiagnosed tumours would have regressed spontaneously without treatment. ${ }^{2,10}$ In addition, screening substantially increases the number of mastectomies performed, ${ }^{2,3}$ despite routine claims to the contrary by advocates of screening.

The best method we have to reduce the risk of breast cancer is to stop the screening program. This could reduce the risk by one-third in the screened age group, as the level of overdiagnosis in countries with organized screening programs is about $50 \% .^{11}$

If screening had been a drug, it would have been withdrawn from the market. Thus, which country will be first to stop mammography screening?

\section{References}

1. Canadian Task Force on Preventive Health Care. Recommendations on screening for breast cancer in average-risk women aged 40-74 years. CMAJ 2011;183:1991-2001.

2. Jørgensen KJ, Keen JD, Gøtzsche PC. Is mammographic screening justifiable considering its substantial overdiagnosis rate and minor effect on mortality? Radiology 2011;260:621-27.

3. Gøtzsche PC, Nielsen M. Screening for breast cancer with mammography [review]. Cochrane Database Syst Rev 2009;(1): CD001877.

4. Gøtzsche PC, Jørgensen KJ, Zahl PH, et al. Why mammography screening hasn't lived up to expectations from the randomised trials. Cancer Causes Control. In press.

5. Jørgensen KJ, Zahl PH, Gøtzsche PC. Breast cancer mortality in organised mammography screening in Denmark. A comparative study. BMJ 2010;340:c1241.

6. Autier P, Boniol M, LaVecchia C, et al. Disparities in breast cancer mortality trends between 30 European countries: retrospective trend analysis of WHO mortality database [published erratum appears in BMJ 2010;341:c4480. LaVecchia, Carlo [corrected to La Vecchia, Carlo]. BMJ 2010;341:c3620.

7. Autier P, Boniol M, Gavin A, et al. Breast cancer mortality in neighbouring European countries with different levels of screening but similar access to treatment: trend analysis of WHO mortality database. BMJ 2011;343:d4411.

8. Bleyer A. US breast cancer mortality is consistent with European data. BMJ 2011;343:d5630.

9. Nyström L, Rutqvist LE, Wall S, et al. Breast cancer screening with mammography: overview of Sweidish randomised trials. Lancet 1993;341:973-8.

10. Zahl PH, Gøtzsche PC, Mæhlen J. Natural history of breast cancers detected in the Swedish mammography screening program; a cohort study. Lancet Oncol 2011 Oct. 11 [Epub ahead of print].

11. Jørgensen KJ, Gøtzsche PC. Overdiagnosis in publicly organised mammography screening programmes: systematic review of incidence trends. BMJ 2009;339:b2587.

Affiliation: Peter C. Gøtzsche is with the Nordic Cochrane Centre, Copenhagen, Denmark. 